\title{
Genetically determined obesity in Prader-Willi Syndrome: the ethics and legality of treatment
}

\author{
Anthony J Holland and Josephine Wong University of Cambridge, Cambridge
}

\begin{abstract}
A central characteristic of people with Prader-Willi

Syndrome (PWS) is an apparent insatiable appetite leading to severe overeating and the potential for marked obesity and associated serious health problems and premature death. This behaviour may be due to the effects of the genetic defect resulting from the chromosome 15 abnormalities associated with the syndrome. We examine the ethical and legal dilemmas that can arise in the care of people with PWS. A tension exists between a genetic deterministic perspective and that of individual choice. We conclude that the determination of the capacity of a person with PWS to make decisions about his/her eating behaviour and to control that behaviour is of particular importance in resolving this dilemma. If the person is found to lack capacity, the common law principles of acting in a person's "best interests" using the "least restrictive alternative" may be helpful. Allowing serious weight gain in the absence of careful consideration of these issues is an abdication of responsibility.
\end{abstract}

(Fournal of Medical Ethics 1999;25:230-236)

Keywords: Capacity; consent; obesity; Prader-Willi Syndrome; satiety

\section{Introduction}

Prader-Willi Syndrome (PWS) is a genetically determined disorder that affects approximately one in 14,000 people of both genders and all ethnic groups. The majority of people with PWS have a small deletion of part of the long arm of chromosome 15 of paternal origin (15q11-13). ${ }^{1} \mathrm{~A}$ central feature of the syndrome is an excessive appetite, with overeating behaviour apparent as early as two years of age. In addition, people with PWS usually require special help at school, due to the presence of mild learning disabilities, as well as having short stature and other phenotypic characteristics. ${ }^{2}$

A proposed pathophysiological explanation for the overeating behaviour is hypothalamic dysfunction ${ }^{3}$ with failure of the feedback mechanism which would normally lead to feelings of fullness and loss of feelings of hunger after eating Hence people with PWS continue to feel hungry even after just eating and therefore continue to consume food and, particularly when unsupers vised, may eat large amounts, giving rise to severe obesity and associated increased morbidity and mortality. Concerns about obesity in people wit PWS go beyond mere aesthetics. Food-seekin behaviour and the weight increase can bE extreme. ${ }^{5}$ For example, carers describe behavioure such as food-stealing, scavenging and eating rave or frozen food. People with PWS can reach such high weights that they may develop severe leg ulcers and limitations in mobility, heart failure diabetes mellitus or other adverse effects of severs obesity, which limit their quality of life and are detrimental to their health. ${ }^{6}$ The life expectancy of people with PWS is likely to be related to how successfully weight is controlled. Appetite sup? pressants, behaviour modification techniques and surgical interventions have all been used to try t $\widehat{B}$ limit food intake but these have had only limite success and at present there are no specific treatio ments for the failure of satiation and overeating behaviour. Dykens et al describe the overeating behaviour as "physiological not motivational" making the point that this is a strongly biologicall determined behaviour and not something that añ individual is able to exert control over. However, ideally with the full consent of the persog concerned, control of access to food and strices supervision at meal times can be effective in pre? venting overeating and is the strategy advocate 4 by PWS support groups in many countries.

The key questions raised are a) does an adult with PWS have the right to determine his/her owf eating behaviour and weight, b) under what, any, circumstances would "compulsory dieting be ethically and legally defensible and c) whaf responsibility do family or paid carers have for controlling access to food and thereby preventing potential life-threatening obesity. Consideration of such issues raises interesting parallels and coris trasts with the wider ethical issues concerning eat- 
ing disorders and obesity. People with PWS may also have an intellectual impairment and mild learning disabilities. As a result, there may also exist the more general tension between the rights of an adult to make decisions about his/her own life and the need for care and protection in the case of some people with learning or other mental disabilities.

\section{Clinical examples}

The following description is typical of the very real dilemmas faced by people with PWS, their families, other carers and health professionals. Ms RL was diagnosed as having PWS by a paediatrician when aged ten, after her parents read an article in a local magazine about the syndrome and asked for a referral. She was subsequently found to have the characteristic deletion on chromosome 15. As a result of their understanding of the syndrome her parents restricted their daughter's access to food. She lost weight, and although her parents felt she was still marginally obese, her weight was considered acceptable. Ms RL was pleased with the outcome, as she was no longer teased at school. At aged 19 she left special school, decided to attend adult education classes at the local college, and started spending periods living away from home at a local group-home for adults with learning disabilities. Her parents had informed the staff about PWS and the risks of obesity. After six months Ms RL's weight had increased from 76 to 89 kilograms and she was raiding the kitchen at the group-home and stealing food at college. When her parents confronted her about this she became very angry and refused to come home. Living permanently at the group-home she continues to put on weight and when seen six months later weighed 115 kilograms. She is breathless, irritable and has ulcers on her legs due to severe swelling of her ankles. She insists she is dieting. The staff feel unable to do any more as locking food away is considered an infringement of RL's "choice" to eat when she wants.

The second example illustrates how the issues go beyond PWS itself and need to be seen in the context of the wider issues of how obesity and obese people are viewed in Western society. In a well-publicised recent case from the United States, ${ }^{8}$ a 13-year-old girl died weighing 309 kilograms, with numerous skin ulcers on her body. The circumstances leading to her death became public knowledge after her mother was initially prosecuted for felony child abuse and was eventually convicted of a lesser crime of misdemeanour child abuse. The question of whether the girl did or did not have PWS was raised after she had died. From the CNN report, which is an example of the broad media coverage of this case, it would seem that much of the controversy surrounding this was raised by the "fat acceptance" or "size acceptance" lobby, questioning society's views on obesity, suggesting that certain assumptions were made about the child and her mother because the child was severely overweight, with the result that neither the girl nor her family received appropriate help. It would seem from the transcript of the discussion programme that the young girl had many but not all the characteristics of PWS. Although her mother had sought help on numerous occasions, no satisfactory explanation was given for her daughter's severe obesity, which had first became apparent early in her daughter's life.

\section{To intervene or not?}

In the case of children with PWS, those with parental responsibility have a clear legal duty of care. If the control of access to money and food is necessary to prevent overeating and resultant severe obesity, it is likely to be legally justifiable and even required as part of one's parental duty to act in one's child's best interest and to protect him/her from harm until he/she reaches adulthood. However, the right of an adult (generally taken to be a person 18 years or older) to autonomy in decision making is central to any democratic society, provided that the person is appropriately informed, has the capacity to make such a decision and is free from undue influence. Health professionals are required to gain consent from a competent adult before proceeding with treatment and to proceed with that treatment without consent may be construed as an assault or battery. In England and Wales, common law provides the framework for intervention when an adult lacks capacity (at present the subject of proposed new legislation ${ }^{9}$ ) and, where the treatment of a mental disorder is required, the Mental Health Act, 1983 (MHA) ${ }^{10}$ may apply.

For people with PWS, adult life results in increasing independence, more unsupervised access to food and therefore a high risk of serious and life-threatening obesity. This could be prevented by close supervision and food restriction. Whilst education about healthy eating and development of treatment strategies that have the person's consent would be the most desirable, often these are not possible because the person with PWS does not wish to consent to the proposed limitations or is unable to keep to them. The question then arises as to whether intervention without the adult's consent is appropriate legally and ethically and, in particular, what action might be taken if the person's life is at risk due to the severity of the obesity. 
Given the present knowledge of PWS we have proposed the following conceptual models for understanding and seeking a resolution to this issue in the context of English and Welsh law. The general principles outlined below will be applicable regardless of the jurisdiction, however, the potential for lawful intervention may differ across countries. We believe that these conceptual models need to be debated because understanding the issues may help resolve what at present are ethical and legal uncertainties.

\section{INDIVIDUAL CHOICE}

One way to conceptualise this dilemma is to consider people with PWS no differently from those in the general population, and more specifically, no differently from people who are overweight. Obesity, with all its associated health problems, is endemic in Western society. In the UK, a reduction in obesity has been one of the targets for the "Health of the Nation" campaign. As humans we appear constitutionally ill-prepared for an environment where food and the money to buy food are readily available. However, it is for each of us as individuals to decide what we eat, how much we eat, the weight we wish to be and the efforts we are prepared to make to attain and maintain our ideal weight. Governments and health care professionals can inform and encourage but cannot insist. No matter how certain we are of the health implications of obesity, as a society we would not sanction regulation or restriction of the food intake of people whose health is at risk, without their consent. This would be considered an unacceptable infringement of civil liberties.

Is overeating behaviour in PWS an extension of the same behaviour so common in the general population? Although people with PWS often have mild learning disabilities and may require special help at school, some may well have average intellectual ability, and the majority have good language ability and are able to make their wishes known. The fact that the majority of people with PWS are able to make quite complex choices about their lives may suggest that they are able to make some choices about their weight and may be prepared to accept the risks and limitations associated with severe obesity. In this model the presence of a genetic disorder and the reported lack of satiety specific to this syndrome are irrelevant. Many people stop eating before they feel completely full for health or aesthetic reasons and this is a choice also available to people with PWS.

It can also be argued that overeating in PWS is no different from common addictive behaviours, for example, to alcohol or tobacco. The person with the addiction understands its adverse effects, but continues to use the substance, often due to라 physical or psychological dependence. Despite the perceived self-destructive nature of these behav iours, our society again does not sanctiobs intervention without the consent of the persob concerned. The principle of autonomy is parass mount even if the outcome is likely to include serious health consequences and/or early death. If we adopt this conceptual approach, adults with PWS should be free to eat as much as they like and intervention to prevent obesity without consen would not only be an infringement of the rights os that person, but also unlawful.

\section{PWS: A SPECIAL CASE OF 'DECISION MAKING CAPACITY' AND/OR 'CONTROL'}

In the case of RL and of others with PWS the central and determining issue is the individual's capacity to make decisions about food intake. If $\mathrm{RL}$ does have the capacity to make such decisions $\frac{\overline{\mathrm{V}}}{\mathrm{V}}$ her decision must be respected even if it leads to marked weight gain and its associated healtko problems. On the other hand, if she is found to lack capacity to make these decisions, under the common law jurisdiction in England and Wales carers would be allowed, and also under a duty, t $\bar{\varnothing}$ act in her "best interests", tive alternative. Failure to supervise access to foo $\$$ in such a case may result not only in dir $\overrightarrow{\hat{E}}$ consequences for the person with PWS, but also reflects negligence on the part of the carers.

The key question when assessing RL's capacity is whether she has the requisite abilities to make this specific decision. The outcome of the decisior (ie, to accept or reject restrictions on access to food) and the level of her intellectual ability' disability by themselves are not determinative o $\mathbb{B}$ her capacity status. To be deemed capable to make decisions about food, RL would have to be able to understand and retain the information that she was seriously overweight and was continuing tô gain weight, that this was a result of her eating more food than her body required and the outcome was that she would be at increasing riski for serious physical illness and might well die. She needs to be able to believe the above informatiof and be able to balance that information to arrive at a choice..$^{12}$

If $R L$ were unable to understand, retain an believe information about healthy eating and the risks of overeating as relating to herself, she would be deemed incapable of making a decision abou? her food intake, therefore carers would be justified in limiting and controlling her access to foodo Each of these need to be considered in turn. Fox example, whilst RL may well be able to under흘 stand and retain the relevant information, her 
insistence that she is in fact dieting, contrary to other evidence, might indicate she does not appreciate that these issues apply directly to her and her wellbeing. A further consideration would be whether or not she is able to balance the relevant information in order to arrive at a choice. Given the fact that hunger and thirst exert powerful influences on an individual's thinking and behaviour, as such drives are essential for survival, it may be very difficult properly to balance the short and longer term consequences of any decision. It might therefore be argued that RL's ability to consider information about food intake is overwhelmed by strong biological drives. Although she may have the capacity to make decisions about many aspects of her life, on matters of eating and weight, this capacity is lacking.

To take the capacity argument one step further in regards to PWS, it has been proposed that since people with this syndrome have a specific chromosomal deletion directly related to satiety and thereby eating behaviour, a special case exists. ${ }^{14}$ Overeating in PWS would be considered to be a genetically determined behaviour and as there can be little control over such a strong biological drive intervention would justified even if the person did not consent. At its most basic, it is not simply an issue of understanding but rather one of control. RL may appreciate the equation of calories in and calories out, and may understand the potential risks of obesity but at the same time have a very limited ability to do anything about it. She has the capacity to understand but not the capacity to control the behaviour, or she may be unable to acknowledge she has limited control of her eating behaviour.

There are some parallels between ethical issues surrounding anorexia nervosa and PWS. Simplistically, overeating in PWS may be seen as a "reverse" of the anorexic behaviour. In anorexia nervosa, the persistent and overvalued ideas about body size drive the extreme dieting behaviour, while in PWS the overeating behaviour occurs as a result of a genetic defect that leads to failure of satiety. The issues of capacity in PWS are in some ways similar to the approach to anorexia nervosa. People with anorexia nervosa are often able to function competently in most areas of their lives, except within this "blind spot" regarding food intake. For example, they may be able to understand and retain information relating to their food intake and the adverse effects of their low weight. However, people with anorexia nervosa may not believe or appreciate that the information about food intake applies to them. In anorexia nervosa, insight may be impaired, particularly in cases where body images are distorted, and individuals may see themselves as much bigger than they really are. In the case of people with PWS, the lack of appreciation may be because of an extreme denial about any weight problem or of the ineffectiveness of their dieting behaviour. Often, as in the case of RL, whilst people with PWS may try to diet their weight continues to increase because the calorie intake is still excessive for their calorie expenditure and they are unable to accept this fact. In both PWS and anorexia nervosa it is both these aspects relating to capacity and control as well as the extreme and life-threatening nature of the behaviour that makes PWS and anorexia nervosa stand out as different from "normal" obesity and "normal" dieting, respectively.

It has been argued that certain people with anorexia nervosa are able to make a decision about food intake. They refuse food not as a symptom of the disorder, but because the quality of life with anorexia is not good enough to outweigh the burden of the treatment. Even if this may result in their death, Draper ${ }^{15}$ argues that their wishes have to be respected. Similarly, if $R L$ has the requisite abilities to make a decision about food intake, but holds the view that since she was born with this disorder and prefers to live with the consequences of obesity rather than the restrictions imposed by others, this may have to be respected.

Adoption of the capacity approach implies that each case has to be taken on its own merit, depending on the ability of the person to make specific decisions about eating. There may be some people with PWS who may never have the capacity to make decisions about their eating, while others may be deemed capable, especially if they have been educated about diet and strategies to reduce weight gain, such as exercising. In those thought to lack capacity, preventing access to food, for example by locking the kitchen or the refrigerator, would be justified under the "best interests" principle.

\section{PWS, A MENTAL DISORDER}

The question of whether PWS can be conceptualised as a mental disorder is significant because many countries have mental health legislation that provides legal means for the compulsory treatment of specific mental disorders. In addition, it may be relevant that anorexia nervosa is now accepted as a mental illness, therefore compulsory treatment may be justified under the Mental Health Acts pertaining if other statutory conditions are satisfied. Whether compulsory intervention is an appropriate and possible course of action will critically depend on the nature of the 
mental health legislation and definitions of what is and what is not included as a mental disorder in such legislation.

We considered issues surrounding PWS in the context of the English and Welsh Mental Health Act, 1983. If PWS and the associated behaviours are considered a "mental disorder" as defined in this act and the person satisfies other conditions of detention under the act, assessment and treatment for the mental disorder in hospital can be carried out without the person's consent. In this situation the question of capacity does not necessarily have to be addressed. A "mental disorder" is broadly defined in the MHA (section 1(2)) as "mental illness, arrested or incomplete development of mind, psychopathic disorder and any other disorder or disability of mind". The presence of early developmental delay and learning disabilities in people with PWS is usually sufficient for them to be said to have "arrested and incomplete development of mind". It might also be argued that as the problem of overeating relates to the failure to feel satiated at a conscious level, people with PWS could therefore be said to have "a disability of mind". If either of these arguments is accepted and other criteria are met, $\mathrm{RL}$, for example, could be admitted under section 2 of the MHA for assessment, close supervision and the imposition of a compulsory diet, as part of her treatment for the consequences of her "disability of mind". However, the assessment period is limited to 28 days. For treatment to be given to $\mathrm{RL}$ for longer and without her consent, a section 3 order would be required. In that case, the definitions of mental disorder are much tighter and she would have to meet criteria for one of four possible categories: mental illness, psychopathic disorder, mental impairment or severe mental impairment. The category most likely to apply is that of "mental impairment", but under these circumstances a significant impairment of intelligence needs to be demonstrated (usually taken to be an IQ of $<70$ using established tests), as well as impairment of social functioning and abnormally aggressive or seriously irresponsible conduct (section 1(2)). This is not always the case with people with PWS.

Even if PWS is considered to fall within the definition of a "mental disorder" or a category of mental disorder, there are major hurdles that may prevent the MHA from being used in controlling access to food in PWS. First, assessment and treatment sections of the MHA can only be used in hospitals or registered mental care homes and cannot be continued for any length of time out of these settings. Secondly, the extent to which the MHA is applicable to a literally life-long condition such as PWS is controversial. Finally, it can be

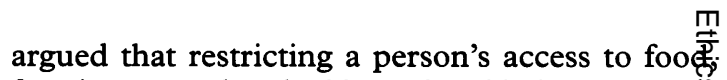
for instance by locking the kitchen or the refrigerator, is not clearly "medical treatment f(함 the mental disorder", even if this is widely defined in the act to include nursing and "care, habilite tion and rehabilitation under medical super sion". An alternative is the use of guardiansh要 orders which offer some limited powers of supew vision in a community setting but the necessary criteria would need to be met.

\section{Conclusions}

The scenario outlined raises significant ethic: dilemmas and legal questions. On the one hand we recognise the rights of people who are able to be autonomous to make decisions for themselves especially with regards to something as funda mental as what and how much they eat. On the other hand, the life-threatening nature of the behaviour provokes the reaction: "something must be done", especially as people with PW may be vulnerable in other ways because of inted lectual impairments. The conceptual models we have proposed illustrate this tension in balancing beneficence and respect for autonomy. Genet determinists might argue that the behaviour of eating is so critical to survival that the breakdow in the physiological control mechanism of the body would have a major effect that woul override any "free will". If the "satiety" model $\overrightarrow{\text { Ff }}$ the eating behaviour and the failure of norma feedback is correct, the constant desire to seek food observed in people with PWS is understan able in terms of a drive that ensures survival.

At the other extreme is the view that overeating in PWS represents the person's free choice, jus like people in the general population who are addicted to alcohol, tobacco or who have difficup ties controlling their weight. There are no grounds for intervention to prevent people from engagin in behaviours that are harmful to themselves, so there should be no justification for intervening to restrict food intake in the person with PWS However, the analogy with dependence on alcohol or tobacco cannot be sustained as these are ne central to life and complete abstinence from alc 6 ? hol or tobacco is certainly possible. The same not true for food.

In response to the questions we raised in the introduction to this paper we propose that PWS a special case and that people with PWS may nơ have the capacity, and therefore the right, to determine their own eating behaviour. Interven tion to prevent obesity, even if the person does nof consent, is likely to be ethically defensible with common law or mental health legislation beir used to enable such a course of action whent 
appropriate. The carer and/or health care professional has a responsibility to determine if intervention is justified through careful consideration of the following; a) the person's capacity to make such decisions, b) knowledge of the person's past eating behaviour and his/her ability to control such behaviour and c) an understanding of the person's past wishes and views about weight and access to food. Given this knowledge the degree of restriction imposed by the intervention should be guided by the extent to which the person with PWS is able to make a decision about his/her eating behaviour and to control it. Central to the intervention that might follow is the principle of "least restrictive alternative". For example, the Department of Health and Welsh Office Mental Health Act Code of Practice refers to the delivery of "any necessary treatment or care in the least controlled and segregated facilities practicable" 16 and specifically in the case of younger people it should be as least restrictive as possible. ${ }^{17}$ The task is to develop a strategy which is both reasonable and practicable in a community setting and where the person with PWS is likely to have periods of independence.

Crucially, knowledge about the eating behaviour of people with PWS in general should ensure that there is a clear and potentially life-long strategy in place to help prevent excessive eating and severe weight gain. ${ }^{18}$ However, precisely what form this takes should be based on an understanding of the strengths and weaknesses of the individual $\mathrm{him} / \mathrm{herself}$. Interventions should include education about healthy eating for people with PWS from childhood. This may be valuable in the long term to enable people with PWS to make at least a partially informed choice to eat healthier foods like vegetables and fruits, or to limit their food intake with or without the help of others. They should be encouraged to express their views as regards what intervention, if any, they would agree to should they become seriously overweight. Monitoring of weight and appropriate positive reinforcements for not gaining or for losing weight and meeting particular weight targets are preferable to "punishment" for gaining weight. Under these circumstances simple rules of supervision and environmental modifications may well be possible and acceptable with beneficial effects and the common law principles of "best interests" and "least restrictive alternative" will have been adhered to. However, there must be a clear appreciation of the very real danger of severe obesity and increased mortality. If severe and lifethreatening obesity does occur, it is not adequate to dismiss the possibility of any further intervention simply on the grounds that it is an individu- al's choice. There is a great risk of considerable harm being done, including the possibility of death, as a result of a misguided commitment to respecting an individual's choice when the capacity to make that choice has not been properly considered and indeed, may well be impaired. Carers and health professionals have a duty very rigorously to consider the issues as described in this paper and to make a reasoned argument in support of whatever course of action is chosen. We suggest that any decision to impose more severe restrictions is not something that rests with one person alone but should involve, as far as possible, the person him/herself, and, if appropriate, relevant family members and appointed advocates and members of relevant disciplines. If such a course of action is strongly opposed by the person with PWS he/she should be supported in getting legal advice and, depending on the circumstances, judicial review or appeal to a Mental Health Review Tribunal can take place. More severe restrictions are likely to include locking of food cupboards and the fridge, and possibly the kitchen door, together with limitations on the amount of money the person with PWS has access to at any one time. Good dietectic advice is crucial to ensure appropriate nutrition, especially if a diet as low as 800 kcals a day is required, as may be the case when someone is severely obese.

Whilst eating behaviour and weight is clearly a matter of choice for the majority of the population we have argued that such an approach must not be applied uncritically. As other genetically determined causes of obesity are described the same issues will need to be examined. For example, in a recently reported case, members of a family with severe obesity were found to have a mutation of the leptin gene, ${ }^{19}$ resulting in a failure of the normal feedback mechanism whereby body mass influences eating behaviour. Would interventions to restrict food intake in such a family be justified?

\section{Acknowledgements}

The views in this paper are those of the authors and we accept responsibility for them, faults and all. However, we would like to thank those with PWS, their parents and other carers and colleagues who have helped us consider these issues. One of the authors (AJH) has recently accepted the offer to become President of the UK PWS Association and would particularly like to acknowledge the work of the association throughout the UK. We would also like to thank Robbie Patterson for her administrative help with the preparation of this paper and Bonnie Kemske for advice on the manuscript. 
Dr Anthony $\mathcal{f}$ Holland, BSc, MBBS, MRCP, $M R C P s y c h$, is a University Lecturer in the Department of Psychiatry, University of Cambridge and Honorary Consultant Psychiatrist, Lifespan NHS Trust, Cambridge. Dr fosephine Wong, MBBS, $M A, M R C P s y c h$, was until recently a Clinical Research Associate at the Department of Psychiatry, University of Cambridge, funded by a grant from the Nuffield Foundation. She is now Lecturer, Department of Psychiatry, University of Hong Kong. Correspondence: Dr A $\mathcal{F}$ Holland, Section of Developmental Psychiatry, Douglas House, $18 b$ Trumpington Road, Cambridge, $C B 22 \mathrm{AH}$.

\section{References}

1 Ledbetter DH, Riccardi VM, Airhart SD, Strobel RJ, Keenan $\mathrm{SB}$, Crawford JD. Deletions of chromosome 15 as a cause of the Prader-Willi Syndrome. The New England fournal of Medicine 1981;304:325-9.

2 Laurance BM. Prader-Willi Syndrome. Pediatric Reviews and Communications 1993;7:77-91.

3 Swaab DF, Purba JS, Hoffman MA. Alterations in the hypothalamic paraventricular nucleus and its oxytocin neurons (putative satiety cells) in Prader-Willi Syndrome: a study of five cases. fournal of Clinical Endocrinology and Metabolism 1995;80:573-9.

4 Holland AJ, Treasure, J, Coskeran P, Dallow J. Characteristics of the eating disorder in the Prader-Willi Syndrome: implications for treatment. Fournal of Intellectual Disability Research $1995 ; 39,5,: 373-81$.
5 See reference 4: 380 .

6 Greenswag LR. Adults with Prader-Willi Syndrome: a surveळ of 232 cases. Developmental Medicine and Child Neurology 198Z 29:145-52.

7 Dykens EM, Goff BJ, Hodapp RM, Davis L, Devanzo P, Mos F, et al. Eating themselves to death: have "personal rights" gon too far in treating people with Prader-Willi Syndrome? Ment Retardation 1997;35:312-14.

8 CNN transcript. Talkback live: the obesity trial. 1997 Dec 3P Web site address: http://www.size-acceptance.or cnn_transcript.htm

9 Lord Chancellor's Division. Who decides? Making decisions oफ behalf of mentally incapacitated adults. (Cm 3803) London: The Stationery Office Limited, 1998

10 Department of Health. Mental Health Act, 1983. Londoñ. HMSO, 1983.

11 Re: F (Mental Patient: Sterilisation) [1990] 2 AC 1.

12 Re: C (Refusal of Treatment) [1994] 1 Family Law Report, 3 P

13 Wong JG, Clare ICH, Gunn MJ, Holland AJ. Capacity to make health care decisions: its importance in clinical practicer Psychological Medicine (in press).

14 See reference $7: 312$.

15 Draper H. Treating anorexics without consent: some reserv tions. Fournal of Medical Ethics 1998;24:5-7.

16 Department of Health and Welsh Office. Mental Health A음 Code of Practice. London: HMSO, 1993:1, para 1.3.

17 See reference $16: 114$, para $30.2 \mathrm{c}$.

18 Holland AJ. The nature of the eating disorder in PWS. Fourn of Applied Research in Intellectual Disability 1998;11:192-206. (D)

19 Montague CT, Farooql IS, Whitehead JP, Soos MA, Rau H Wareharn NJ, et al. Congenital leptin deficiency is associate with severe early-onset obesity in humans. Nature 1997;38 $902-8$.

\section{News and notes}

\section{The Annual Intensive Course on Medical Ethics}

The Annual Intensive Course on Medical Ethics will take place at Imperial College, London from 13-17 September, 1999.

The course provides a multidisciplinary introduction to philosophical medical ethics for medical and nursing teachers, members of ethics committees, GPs, hospital consultants and health administrators.
It is organised in collaboration with the Institute of Medical Ethics.

For further information please contact: Sally Verkaik, Imperial college Centre for Continuing Education, London SW7 2AZ. Tel: +44 (0) 171594 6882; fax: +44

(0)171 594 6883; e-mail: cpd $\omega$ ic.ac.uk

\section{News and notes}

\section{Human Rights in Medicine}

Human Rights in Medicine is the title of an international summer course on health care ethics to be held from June 28 - July 3 in Dubrovnik, Croatia.

For further information please contact: Miroslav
Mastilica, PhD, Andrija Stampar School of Public Health, University of Zagreb, Rockefellerova 4, HR10000 Zagreb, Croatia. Tel: +385 1468 4440; fax: +385 1468 4441; email: mmastil $a$ andrija.snz.hr 